Matamoros-Calderón, W., Lara, M., \& Breedy, O. (2021). Bosques de coral negro del Área de Conservación Guanacaste: especies y distribución. Revista de Biología Tropical, 69(Suppl. 2), S208-S218. https://doi.org/10.15517/rbt. v69iS2.48317

\title{
Bosques de coral negro del Área de Conservación Guanacaste, Costa Rica: especies y distribución
}

Wendolyn Matamoros-Calderón ${ }^{1,2}$; (D) https://orcid/org/0000-0002-2715-1875

Minor Lara ${ }^{3}$; (D https://orcid/org/0000-0002-1571-2700

Odalisca Breedy $1,2,4,5$; (D) https://orcid/org/0000-0001-5686-4164

1. Centro de Investigación en Estructuras Microscópicas (CIEMIC), Universidad de Costa Rica, 11501-2060, San José, Costa Rica; wemaca92@gmail.com

2. Centro de Investigación en Ciencias del Mar y Limnología (CIMAR), Universidad de Costa Rica, 11501-2060, San José, Costa Rica; odaliscab@gmail.com

3. Diving Center Cuajiniquil, La Cruz, Guanacaste, Costa Rica; minorlara@hotmail.com

4. Museo de Zoología, Centro de Investigación en Biodiversidad y Ecología Tropical (CIBET), Universidad de Costa Rica, 11501-2060, San José, Costa Rica.

5. Smithsonian Tropical Research Institute, Ciudad de Panamá, República de Panamá.

Recibido 30-I-2021. Corregido 07-V-2021. Aceptado 02-VI-2021.

\section{ABSTRACT \\ Black coral forests in the Guanacaste Conservation Area, Costa Rica: species and distribution}

Introduction: Little is known about the antipatharians in Costa Rica, only three species are known from the Pacific and the occurrence of coral forests was previously reported only for Isla del Coco. Black coral forests were observed at several sites in the north Pacific of the country, within the Guanacaste Conservation Area (ACG), which are reported and characterized in this study.

Objective: To determine the species of black corals present in the ACG Marine Sector and its surroundings, and geographically locate the sites where black coral forests grow.

Methods: Visual censuses were made by diving, samples were taken and analyzed in the laboratory for identification.

Results: These black coral forests are composed of two species: Myriopathes panamensis and Antipathes galapagensis, these species are described and illustrated. The coral forests were found within and outside ACG marine protected areas. Myriopathes panamensis was found between 15-35 m depth and A. galapagensis at 30-45 m. Associated macrofauna was observed among the branches of $M$. panamensis, their ecological importance and status have not been determined.

Conclusions: The species found did not have morphological variations with those described in other similar regions. Associated fauna was found (mainly the anemone Nemanthus californicus, the bivalve Pteria sterna and the longnose hawkfish Oxycirrhithes typus) and needs to be studied to determine its role on black corals. The importance of the ACG as a marine biodiversity conservation site is emphasized.

Key words: Antipatharia; associated fauna; biodiversity; pinnules; taxonomy. 
Los corales negros son organismos sésiles coloniales que pertenecen al orden Antipatharia Milne-Edwards \& Haime, 1857 (Anthozoa: Hexacorallia) y se caracterizan por tener pólipos pequeños que producen colonias con un eje central proteínico, no calcáreo (Bo, Montgomery, Opresko, Wagner, \& Bavestrello, 2019; Opresko, 1976). Son importantes componentes de la estructura tridimensional de los fondos marinos, ya que proveen refugio y hábitat para alimentación, cuido y crecimiento de muchas especies de peces e invertebrados (Etnoyer \& Morgan, 2005; Prouty, Roark, Buster, \& Ross, 2011). La gran cantidad de fauna asociada, que incluye esponjas, anémonas, moluscos y otros grupos, los convierte en reguladores de las comunidades bénticas y el funcionamiento del ecosistema (Bo et al., 2012; Excoffon, Acuña, \& Cortés, 2009; Prouty et al., 2011). Debido a que los corales negros son organismos muy longevos (con reportes de hasta 4265 años) (Love, Yoklavich, Black, \& Andrews, 2007; Roark, Guilderson, Dunbar, Fallon, \& Mucciarone, 2009) y a que tienen una tasa de crecimiento radial muy lenta, son considerados bioindicadores siendo importantes en temas de conservación y cambio climático (Raimundo et al., 2013). Estos organismos se encuentran distribuidos en todo el mundo y la mayoría de las especies se encuentran en zonas mayores a los $50 \mathrm{~m}$ de profundidad (Bo et al., 2012; Bruckner, De Angelis, \& Montgomery, 2008; Brugler, Opresko, \& France, 2013; Wagner, Luck, \& Toonen, 2012). Sin embargo, el grupo se ha visto amenazado por diferentes acciones destructivas como la pesca de arrastre y la extracción con fines comerciales para usos en joyería (Bo et al., 2019; Gori et al., 2019; Grigg, 2001). Debido a esto, fueron enlistados en el Apéndice II de la Convención sobre el Comercio Internacional de Especies Amenazadas de Flora y Fauna Silvestres (CITES).

A pesar de su importancia tanto económica como ambiental, los corales negros han sido poco estudiados a nivel mundial y las costas de América Latina no son la excepción (Bo et al., 2012). En el Pacífico de Costa Rica se ha registrado Myriopathes panamensis (Verrill, 1869),
Antipathes galapagensis Deichmann, 1941 y Lillipathes ritamariae Opresko \& Breedy, 2010 (Brugler et al., 2013; Cortés, 2019; Opresko \& Breedy, 2010; Reimer \& Fujii, 2010). En México, Panamá, Ecuador y Colombia se han reportado dos especies de corales negros que forman bosques; en Costa Rica, solamente se ha reportado la presencia de bosques en el Parque Nacional Isla del Coco (Brugler et al., 2013; Cortés, 2019; Opresko \& Breedy, 2010; Reimer \& Fujii, 2010).

El archipiélago de Islas Murciélago (protegido dentro del Sector Marino) y el Golfo de Santa Elena representan importantes centros de biodiversidad dentro del Área de Conservación Guanacaste (ACG) (Cortés, 2017). En las Islas Murciélago se han observado asentamientos de corales negros formando bosques, cuya existencia no había sido documentada previamente en la literatura científica. También se han observado bosques de coral negro en bajos de pesca que han sido explotados durante muchos años, fuera del área marina protegida. Este estudio presenta las observaciones de las poblaciones de corales negros en aguas someras que se han recopilado durante años de buceo. El objetivo de este trabajo es reportar la ubicación de bosques de coral negro dentro del ACG y describir de forma general su fauna asociada, generando una línea base para futuras investigaciones.

\section{MATERIALES Y MÉTODOS}

Sitio de estudio: El Área de Conservación Guanacaste se encuentra en la zona norte de Costa Rica y cuenta con una extensión marina de 43000 ha y $150 \mathrm{~km}$ de costa que se ubican en varias categorías de protección (ChacónMonge, Azofeifa-Solano, Alvarado, \& Cortés, 2021; Cortés, 2017) (Fig. 1). Este sitio se caracteriza por estar influenciado por los vientos alicios provocando una zona de afloramiento con aguas frías, ricas en nutrientes y oxígeno; lo cual influye en que el ACG albergue una alta diversidad de especies (Chacón-Monge et al., 2021; Cortés, 1996). Además, el ACG es Patrimonio Mundial de UNESCO, lo que recalca 

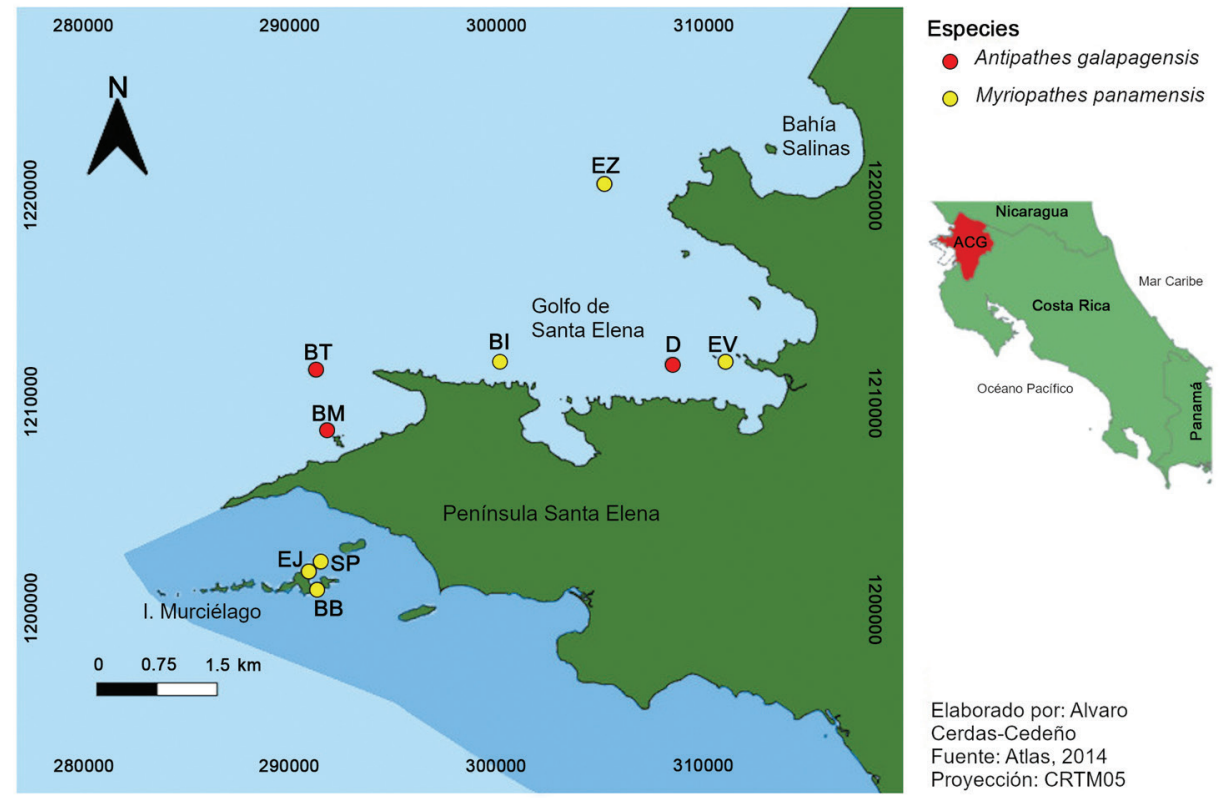

Fig. 1. Localidad de los bosques de coral negro conocidos en el Área de Conservación Guanacaste (ACG) listados en la Tabla 1. El color del círculo indica la especie dominante, y la zona sombreada en azul oscuro indica el área marina protegida (Sector Marino del ACG).

Fig. 1. Location of the black coral forests known in the Guanacaste Conservation Area listed in Table 1. The color of the circles indicates the dominant species, and the dark blue shaded area the marine protected area (Marine Sector of ACG).

su valor en la conservación de los recursos naturales que allí se encuentran (Cortés \& Joyce, 2020).

Metodología: Durante los últimos 20 años se han realizado exploraciones mediante buceo SCUBA en el ACG. Se determinaron 9 puntos donde se encuenran bosques de coral negro, los cuales fueron georeferenciados por medio de GPS (Fig. 1). Durante este tiempo, se hicieron observaciones de los bosques de coral negro presentes en la zona. Por medio de observaciones cualitativas realizadas por una misma persona, se determinó la especie de coral negro dominante en cada sitio, considerándose como dominante la que abarcara la mayor área del bosque, por un mayor número de colonias o porque estas fueran de mayor tamaño. Se elaboró un mapa con el programa QuantumGIS (QGIS Development Team, 2016) usando el Atlas 2014 de Costa Rica (Ortiz-Malavasi,
2014) para reportar la distribución de los corales negros en el ACG.

Después de identificada la especie en el campo, se tomaron muestras de los corales negros para analizarlas en el laboratorio. Para el estudio microscópico, se tomaron fragmentos pequeños de las pínulas y se trataron con hipoclorito de sodio $3 \%$, que fueron analizadas por medio de microscopía electrónica de barrido (MEB Hitachi 3700, 570) para la caracterización morfológica.

Se examinaron las especies de fauna asociada por medio de fotografías de los organismos observados sobre los corales negros y posteriormente fueron identificados al mínimo nivel taxonómico posible corroborando con expertos en los grupos. El conocimiento empírico de buzos locales, tanto de SCUBA como de compresor, fueron importantes para el registro de cambios observados en los corales negros en el tiempo. 
TABLA 1

Profundidad y estado de protección de los sitios del Área de Conservación Guanacaste con bosques de coral negro y especie dominante en cada uno (MP: Myriopathes panamensis, AG: Antipathes galapagensis), Pacífico norte de Costa Rica

TABLE 1

Depth and protection status of the Guanacaste Conservation Area sites with black coral forests and dominant species in each (MP: Myriopathes panamensis, AG: Antipathes galapagensis), North Pacific of Costa Rica

\begin{tabular}{clccc}
\hline Código & \multicolumn{1}{c}{ Sitio } & Profundidad $(\mathrm{m})$ & Estado de protección & Especie dominante \\
\hline EZ & El Zoncho & 35 & No protegido & MP \\
EV & Bajo El Viejón & 15 & No protegido & MP \\
D & Danta & 30 & No protegido & AG \\
BI & Bajo Islita & 30 & No protegido & MP \\
BT & Bajo Tigre & 45 & No protegido & AG \\
BM & Bajo Mogotes & 34 & No protegido & AG \\
SP & San Pedrillo & 32 & AMP & MP \\
EJ & El Jardín & 25 & AMP & MP \\
BB & Bajo Bosque & 31 & AMP & MP \\
\hline
\end{tabular}

\section{RESULTADOS}

Se observaron dos especies de coral negro, M. panamensis (Myriopathidae) y A. galapagensis (Antipathidae), formando estructuras boscosas tridimensionales. Ambas se observaron en sitios dentro y fuera del área marina protegida en el Pacífico norte de Costa Rica (Fig. 1; Tabla 1), con una mayor cantidad de bosques dominados por $M$. panamensis que por A. galapagensis. De esta última especie se observaron bosques únicamente fuera de áreas protegidas, a pesar de que sí está presente dentro del área protegida. En general, cada bosque está constituido por una especie dominante, donde se observan algunas colonias dispersas de la especie que no lo es. Aparentemente las agregaciones de las los corales negros están relacionadas con la profundidad, encontrándose $M$. panamensis en aguas más someras, a partir de los $15 \mathrm{~m}$ de profundidad, como se observa en El Viejón (Fig. 1; Tabla 1); mientras que $A$. galapagensis se encuentra a mayor profundidad, formando bosques que llegan hasta los $45 \mathrm{~m}$, según las observaciones que se realizaron en este estudio; sin embargo, los buzos de compresor han observado estos bosques hasta $70 \mathrm{~m}$ de profundidad.

\section{Caracterización de las especies:}

Familia Antipathidae Pallas, 1766 Antipathes galapagensis Deichmann, 1941

Diagnosis (basada en Deichmann, 1941;

Opresko, 2009; Opresko, com. pers.)

Colonias arborescentes, de hasta $1 \mathrm{~m} \mathrm{o}$ más de alto, diámetro del tallo varía entre 0.8-2 cm (Fig. 2A, Fig. 2B). Ramificación densa e irregular (Fig. 2A, Fig. 2B, Fig. 2C), bifurcaciones al décimo orden o más (Fig. 3A). Pinnas y pínnulas alargadas, con apariencia sinuosa, surgiendo de todos lados de las ramas de los órdenes inferiores en ángulos abiertos, uniseriales en algunas partes y con espinas distribuidas longitudinalmente (Fig. 3B). Espinas polipares cónicas, puntiagudas con papilas muy pequeñas y agudas o con estrías poco pronunciadas en la parte apical, con uno o dos lóbulos en el ápice (Fig. 3C, Fig. 3D). Espinas polipares entre $0.12-0.16 \mathrm{~mm}$ de altura, espinas abpolipares entre 0.09-0.14 mm. Pólipos entre 1-2 $\mathrm{mm}$ de diámetro transversal, contorno circular, apertura bucal cónica, rodeado por 6 tentáculos de igual longitud, entre 6-7 pólipos por centímetro en las ramas. Color de los pólipos amarillento o verdoso (Fig. 2A, Fig. 2B, Fig. 2C). 

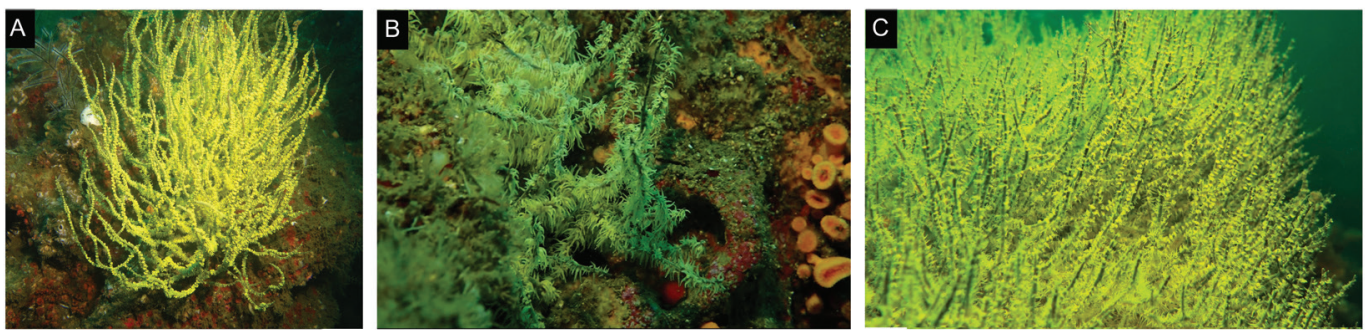

Fig. 2. Colonias de Antipathes galapagensis (Antipathidae) en Bajo Islita, a $30 \mathrm{~m}$ de profundidad. A) Morfología de la colonia; B) detalle de los pólipos; C) vista de la agregación de colonias.

Fig. 2. Colonies of Antipathes galapagensis (Antipathidae) in Bajo Islita at $30 \mathrm{~m}$ depth. A) Colony morphology; B) polyps' detail; C) colony aggregation view.

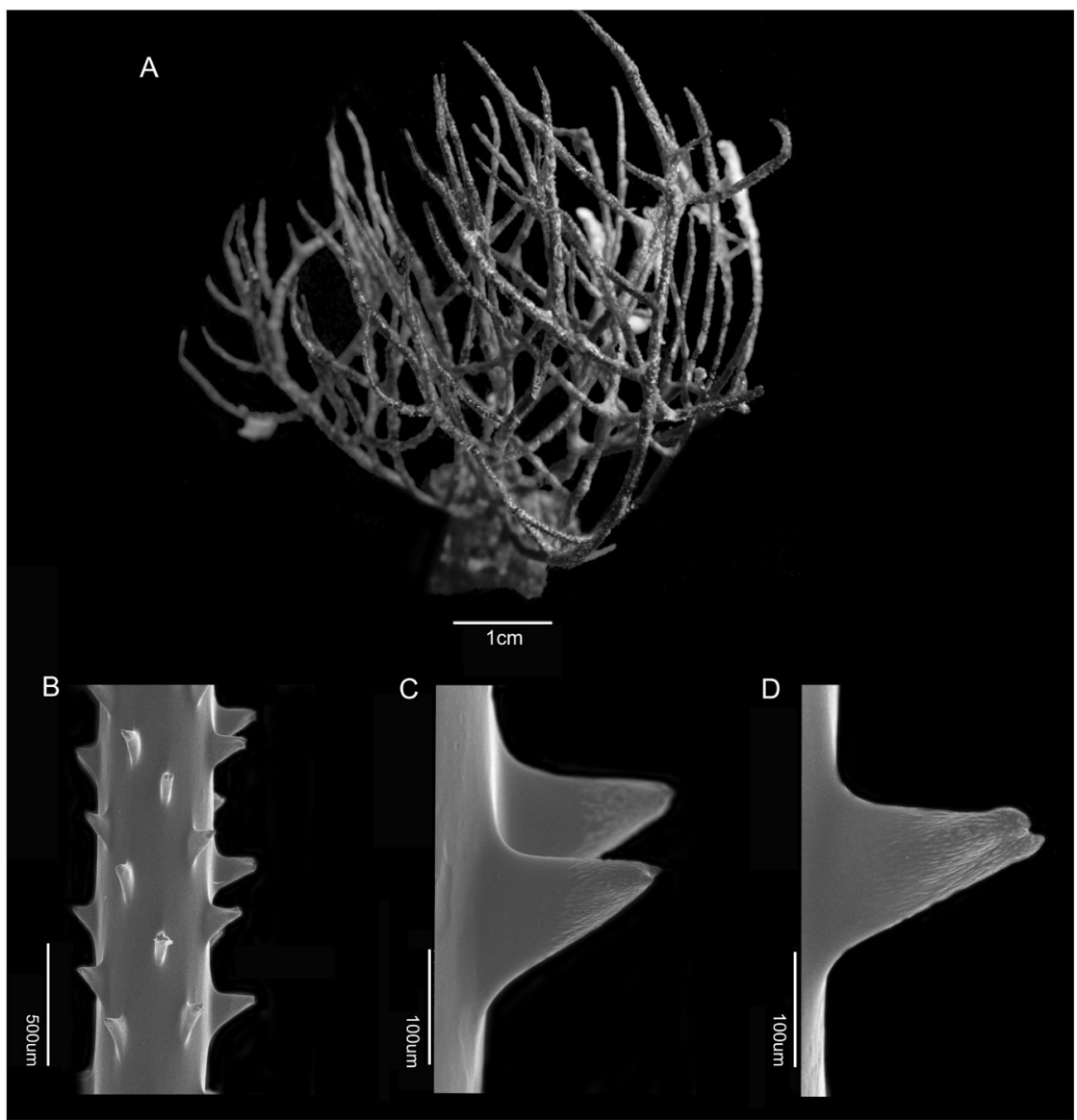

Fig. 3. A) Colonia de Antipathes galapagensis (Antipathidae) preservada (UCR 1802); B) disposición de las espinas en el tallo; C-D) detalle de las espinas.

Fig. 3. A) Preserved colony of Antipathes galapagensis (Antipathidae) (UCR 1802); B) arrangement of the spines on the stem; C-D) detail of the spines. 

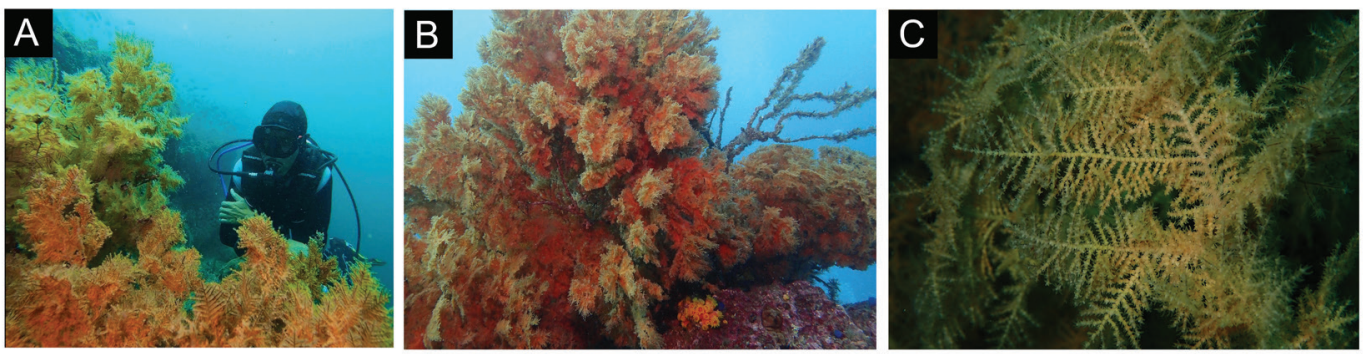

Fig. 4. Colonias de Myriopathes panamensis (Myriopathidae) en El Viejón a 15 m de profundidad. A) Vista del bosque de coral; B) colonias y fauna asociada; C) detalle de los pólipos en las pínulas.

Fig. 4. Colonies of Myriopathes panamensis (Myriopathidae) in El Viejón at a depth of $15 \mathrm{~m}$. A) View of the coral forest; B) colonies and associated fauna; C) detail of the polyps on the pinnules.

Esta especie se puede encontrar en el Pacífico Tropical Oriental, desde el Golfo de California hasta Ecuador (Bo et al., 2012; Wills-López, 2008). Su distribución batimétrica es variable, encontrándose en arrecifes y plataformas rocosas localizadas a profundidades entre 15-90 m (Bo et al., 2012).

Familia Myriopathidae Opresko, 2001 Myriopathes panamensis (Verril, 1869)

Diagnosis (basada en Bo et al., 2012; Brook, 1889; Hickman, 2008; Verrill, 1869; Opresko, 1976).

Colonias arborescentes, hasta $1.50 \mathrm{~m} \mathrm{o}$ más de alto, diámetro del tallo varía entre 0.8-5.9 cm (Fig. 4A, Fig. 4B). Ramificación extendida lateralmente en un mismo plano con un tallo basal corto (Fig. 4C). Pínnulas densas dispuestas bilateralmente (Fig. 5A, Fig. 5B). Pinnas y pínnulas con espinas distribuidas de forma irregular (Fig. 5A, Fig. 5B), estas espinas con la punta curvada hacia arriba o aplanadas hacia el exterior (Fig. 5D). Espinas de las ramas cónicas con ápice redondeado y distribuidas irregularmente (Fig. 5C). Se pueden encontrar de 7-9 filas longitudinales, encontrándose de 7-9 espinas por milímetro por fila, entre los 0.14-0.16 mm de longitud (Fig. 5D). Pólipos entre $0.5-0.7 \mathrm{~mm}$ de diámetro transversal, de 10-12 por centímetro en las ramas. Color de los pólipos blanquecinos alargados con tentáculos cilíndricos (Fig. 4C). Colonias café oscuro (Fig. 4A, Fig. 4B, Fig. 4C). Se ha

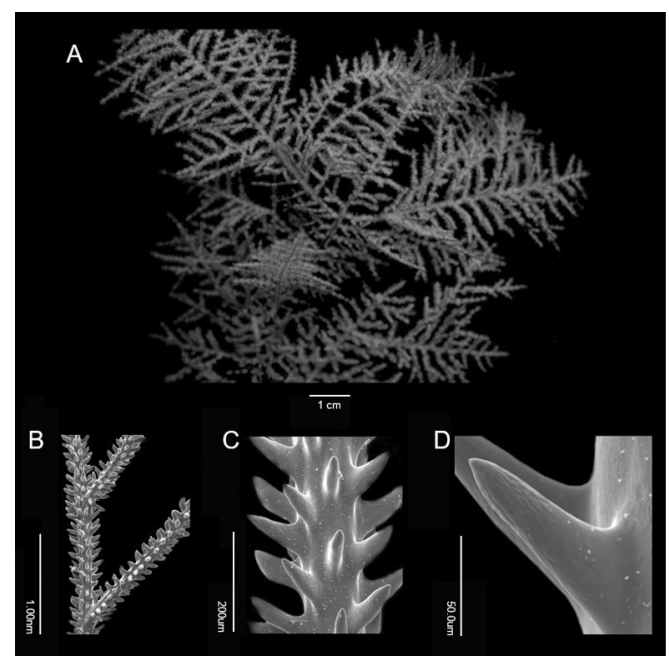

Fig. 5. A) Colonia de Myriopathes panamensis preservada (UCR 1792) y pínulas terminales; B) disposición de las espinas en el tallo; C-D) detalle de las espinas.

Fig. 5. A) Preserved colony of Myriopathes panamensis (UCR 1792), and terminal pinnules; B) arrangement of the spines on the stem; C-D) detail of the spines.

registrado desde México hasta Ecuador en el Pacífico Tropical Oriental y su distribución batimétrica reportada va desde los 9-50 $\mathrm{m}$ de profundidad (Bo et al., 2012).

Fauna asociada: Por la morfología de sus colonias, la fauna asociada se documentó principalmente entre las ramas de las colonias de $M$. panamensis. Sobre este coral negro se observaron organismos como el bivalvo Pteria sterna (Pteriidae), el cual es muy abundante en algunos sitios como El Viejón (Fig. 6A, 

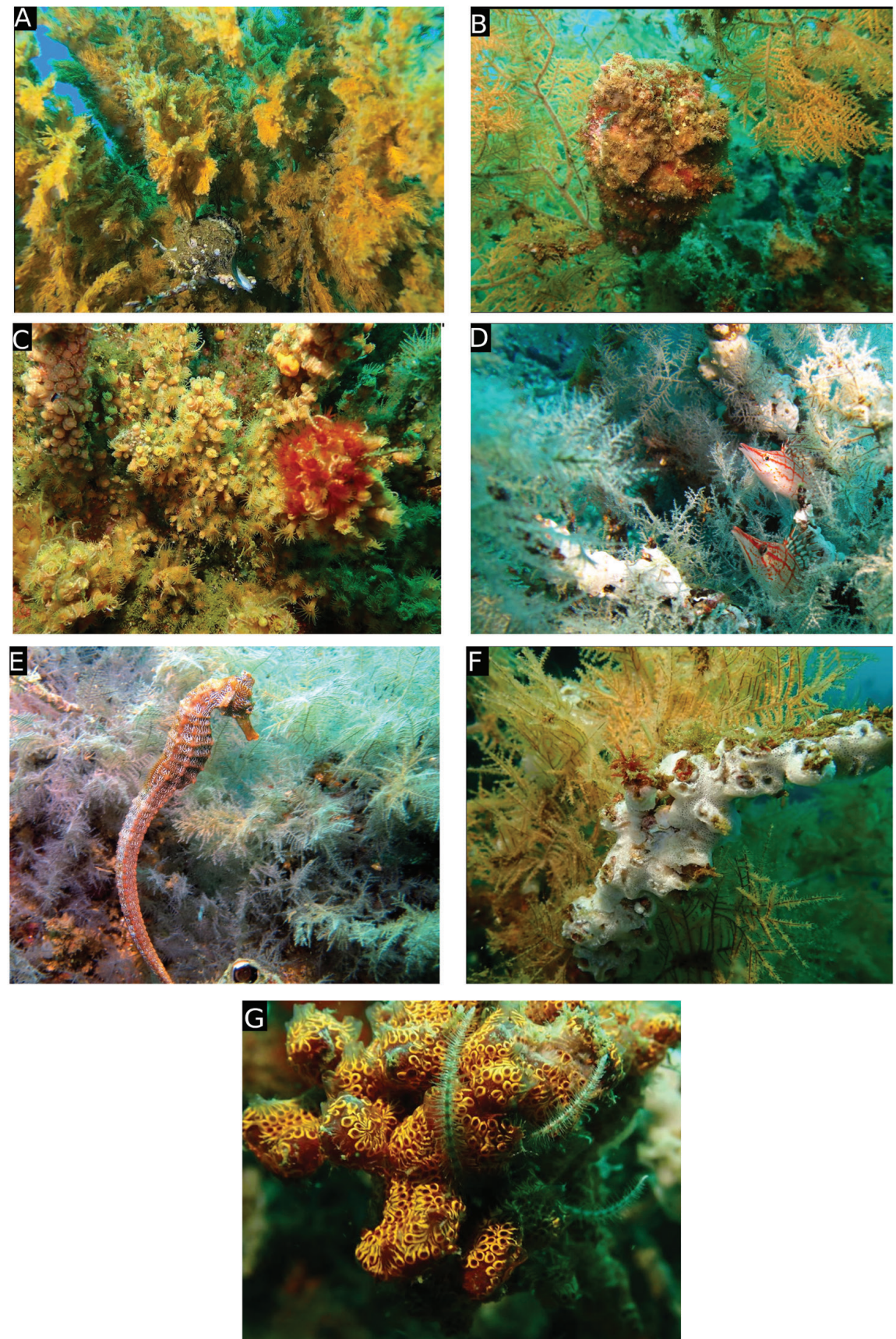

Fig. 6. Fotos submarinas de la fauna asociada a Myriopathes panamensis (Myriopathidae): A-B) el bivalvo Pteria sterna (Pteriidae); C) la anémona Nemanthus californicus (Nemanthidae); D) el pez halcón narigón Oxycirrhites typus (Cirrhitidae); E) el caballito de mar Hippocampus ingens (Syngnathidae); F) una ascidea de la familia Didemnidae; G) la ascidea Botrylloides leachii (Styelidae), y la ofiura Ophiothrix spiculata (Ophiotrichidae).

Fig. 6. Underwater photos of the fauna associated with Myriopathes panamensis (Myriopathidae): A-B) the bivalve Pteria sterna (Pteriidae); C) the anemone Nemanthus californicus (Nemanthidae); D) the longnose hawkfish Oxycirrhites typus (Cirrhitidae); E) the Pacific seahorse Hippocampus ingens (Syngnathidae); F) an ascidean (Family Didemnidae); G) the ascidean Botrylloides leachii (Styelidae), and the brittle star Ophiothrix spiculata (Ophiotrichidae). 
Fig. 6B). Otros organismos como las anémonas Nemanthus californicus (Nemanthidae), se encontraron cubriendo ramas completas de coral negro muerto (Fig. 6C); estas ramas muertas pueden ser colonizadas por esponjas, cirripedios, hidrozoarios y otros organismos. Algunos peces como el halcón narigón Oxycirrhithes typus (Cirrhitidae, Fig. 6D) y caballitos de mar Hippocampus ingens (Syngnathidae, Fig. 6E) también se observaron asociados al coral negro, igualmente ambos se han encontrado en las ramas de octocorales. Además, se observaron ascideas de la familia Didemnidae (Fig. 6F) y otras de la especie Botrylloides leachii (Styelidae, Fig. 6G), así como los ofiúridos o estrellas quebradizas Ophiotrix spiculata (Ophiotrichidae, Fig. 6G). Es necesario un análisis más detallado para determinar la fauna asociada a $A$. galapagensis.

Según las observaciones recopiladas para este estudio, los buzo locales indican que a principios de la década de 1990 los bosques de $M$. panamensis eran más abundantes y los peces $O$. typus eran muy comunes entre sus ramas; sin embargo, para finales de esa década el pez fue extraído con fines decorativos para acuarios y su abundancia disminuyó drásticamente. A inicios de los años 2000 hubo un aumento en las poblaciones de las anémonas y en pocos años ya solo se observaron los esqueletos del coral cubiertos por las anémonas (N. californicus) (Excoffon et al., 2009). En el 2010 los corales negros se habían muerto casi en su totalidad. En algunos de estos sitios, colonias de $A$. galapagensis empezaron a crecer sobre los tallos de los corales muertos de M. panamensis. Un caso parecido ocurrió en bajo El Viejón, donde se extrajo gran cantidad del pez O. typus y en los últimos 10 años una población importante del bivalvo $P$. sterna se ha desarrollado sobre las ramas del coral, quebrándolas en algunos casos. El bivalvo tiene a su vez una gran cantidad de organismos asociados como cirripedios, esponjas, entre otros que aumentan su peso; lo cual necesita ser estudiado ya que podría estar causando mayor incidencia de corales quebrados.

\section{DISCUSIÓN}

Las dos especies observadas en el ACG no tienen variaciones morfológicas respecto a las descritas para otros lugares del PTO (Bo et al., 2012; Hickman, 2008; Opresko, 1976; Opresko, 2009). La presencia de bosques de A. galapagensis fuera del área protegida y la ausencia dentro de áreas protegidas puede deberse a la falta de exploración a más profundidad o a otros factores bióticos (competencia o depredación) y abióticos (temperatura, sustrato o corrientes), que deben ser analizdos en futuros estudios. Se ha propuesto que los efectos causados por el cambio climático pueden afectar en gran medida la composición y abundancia de las comunidades marinas (Bo et al., 2012). Investigaciones anteriores han mencionado que las condiciones óptimas en donde se desarrollan los corales negros se dan por debajo de los $100 \mathrm{~m}$ (Wagner et al., 2012). Gori et al (2019) han informado que cada vez es más común el registro de corales negros a menor profundidad. En este estudio se encontró $M$. panamensis en profundidades desde los $15 \mathrm{~m}$ y $A$. galapagensis desde los $30 \mathrm{~m}$ de profundidad, concordando con lo reportado en la literatura (Bo et al., 2012; Bo et al., 2019).

La macrofauna observada entre las ramas de $M$. panamensis no ha sido evaluada en cuanto a su significado ecológico. La anémona $N$. californicus fue encontrada cubriendo las ramas de corales muertos, en algunos bosques alrededor de las Islas Murciélago como el bajo El Jardín; algunos autores han observado que las anémonas de este género pueden llegar a matar las colonias de coral (Excoffon et al., 2009; Gress \& Kaimuddin, 2021), probablemente para tener mayor disponibilidad de sustrato (Gress \& Kaimuddin, 2021).

Algunos autores mencionan otros organismos como fauna asociada a los corales negros dentro de los que destacan ascidias, esponjas, cirripedios, zoántidos y algas (Bo et al., 2012). Todos estos han sido observados en el Pacífico norte, pero no se ha hecho un estudio taxonómico detallado de los mismos. Vargas (2000) describió una especie nueva de 
camarón de la familia Palaemonidae (Periclimenes murcielaguensis Vargas, 2000) asociada a $M$. panamensis, pero desde entonces no se ha vuelto a observar. Es posible que ésta y otras especies de crustáceos, por su camuflaje y ser escurridizos, no se hayan recolectado recientemente y para encontrarlas sea necesario recurrir a nuevas estrategias de muestreo.

Como se demuestra en este estudio, el ACG alberga parches de bosques de coral negro, que son centros de biodiversidad y que tienen una importancia económica potencial (ej. productos bioquímicos, turismo) que no ha sido evaluada, por lo que se acentúa la importancia de esta región en cuanto a conservación. Los posibles impactos de la extracción no controlada de peces para acuario que se asocian a los bosques de coral negro deben ser estudiados a profundidad. La mortalidad de coral negro observada también debe ser motivo de estudio, por lo que un monitoreo de estos ambientes será fundamental para entender mejor su dinámica. Este trabajo preliminar proporciona una línea de base para estudios ecológicos y taxonómicos de los corales negros, y también sugiere la importancia de un estudio dirigido a evaluar el impacto de $N$. californicus, O. typus y P. sterna sobre las comunidades de corales negros presentes en el ACG. Además, en general, se debe de hacer un esfuerzo para describir la diversidad de corales negros en el país y las interacciones con la fauna asociada.

Declaración de ética: los autores declaran que todos están de acuerdo con esta publicación y que han hecho aportes que justifican su autoría; que no hay conflicto de interés de ningún tipo; y que han cumplido con todos los requisitos y procedimientos éticos y legales pertinentes. Todas las fuentes de financiamiento se detallan plena y claramente en la sección de agradecimientos. El respectivo documento legal firmado se encuentra en los archivos de la revista.

\section{AGRADECIMIENTOS}

Agradecemos a Dennis Opresko por su valiosa ayuda en la identificación de las especies de corales negros y su procesamiento. A Beatriz Naranjo, Rita Vargas, Kaylen González, Leonardo Chacón y Fabian Acuña por la identificación de organismos asociados y a Jorge Cortés por su aporte valioso a nuestras observaciones de campo. A Álvaro Cerdas por su colaboración y apoyo en la elaboración del mapa de sitios del ACG y a Yelba Vega (BioMar-ACG) por los datos de distribución. A Diving Center Cuajiniquil por el apoyo en la recopilación de los datos y fotografías. Este trabajo fue financiado parcialmente por el proyecto B9508: Biodiversidad Marina del Área de Conservación Guanacaste, Costa Rica: BioMar-ACG, de la Vicerrectoría de Investigación UCR y BioMar-ACG Project financiado por Guanacaste Dry Forest Conservation Fund (GDFCF). Nuestro agradecimiento a los revisores anónimos cuyas observaciones fueron muy acertadas para mejorar nuestra publicación.

\section{RESUMEN}

Introducción: El conocimiento de los antipatarios en Costa Rica es muy escaso, se ha informado de tres especies en el Pacífico y de la existencia de bosques de coral en la Isla del Coco. En este estudio se informa de la presencia de bosques de coral negro y sus características encontrados en el Área de Conservación Guanacaste (ACG) sector marino. Objetivo: Determinar las especies de corales negros presentes en el Sector Marino del ACG y alrededores, y localizar geográficamente los sitios en que se desarrollan los bosques de coral negro.

Métodos: Se hicieron censos visuales cualitativos mediante buceo, se tomaron muestras y analizaron en el laboratorio para su identificación.

Resultados: Los bosques de corales negros están compuestos por dos especies: Myriopathes panamensis y Antipathes galapagensis las cuales son descritas e ilustradas. Los bosques de coral se encuentran distribuidos dentro y fuera del Sector Marino del ACG. Myriopathes panamensis se observó entre 15-45 m de profundidad y A. galapagensis entre $30-45 \mathrm{~m}$. Entre las ramas de M. panamensis se 
observó macrofauna asociada cuyo estatus e importancia ecológica no han sido estudiados.

Conclusiones: Las especies encontradas no tuvieron variaciones morfológicas con las descritas en otras regiones similares. Se sugiere la importancia de un estudio dirigido a evaluar el rol de la fauna asociada con los corales negros, principalmente de la anémona Nemanthus californicus, el bivalvo Pteria sterna y el pez halcón narigón Oxycirrhithes typus. Se enfatiza la importancia del Área de Conservación Guanacaste como sitio de conservación de la biodiversidad marina.

Palabras clave: Antipatharia; fauna asociada; biodiversidad; pínulas; taxonomía.

\section{REFERENCIAS}

Bo, M., Lavorato, A., Di Camillo, C. G., Poliseno, A., Baquero, A., Bavestrello, G., \& Reimer, J. D. (2012). Black coral assemblages from Machalilla National Park (Ecuador). Pacific Science, 66(1), 63-81.

Bo, M., Montgomery, A. D., Opresko, D. M., Wagner, D., \& Bavestrello, G. (2019). Antipatharians of the mesophotic zone: four case studies. In Y. Loya, K. Puglise, \& T. Birdge (Eds.), Mesophotic Coral Ecosystems (pp. 465-475). Cham, Switzerland: Springer Nature.

Brook, G. (1889). Report on the Antipatharia. Report of the scientific results of the voyage of the H.M.S. Challenger. Zoology, 32, 1-222.

Bruckner, A., De Angelis, P., \& Montgomery, T. (2008). Case study for black coral from Hawaii. En International Expert Workshop on CITES Non-detriment Findings, Cancun, November 17th-22nd. http:// www.conabio.gob.mx/institucion/cooperacion_internacional/TallerNDF/Links-Documentos/WG-CS/ WG9-AquaticInvertebrates/WG9-CS1\%20BlackCoral/WG9-CS1.pdf

Brugler, M. R., Opresko, D. M., \& France, S. C. (2013). The evolutionary history of the order Antipatharia (Cnidaria: Anthozoa: Hexacorallia) as inferred from mitochondrial and nuclear DNA: implications for black coral taxonomy and systematics. Zoological Journal of the Linnean Society, 169(2), 312-361.

Chacón-Monge, J. L., Azofeifa-Solano, J. C., Alvarado, J. J., \& Cortés, J. (2021). Área de Conservación Guanacaste Echinoderms, North Pacific of Costa Rica. Revista de Biología Tropical, 69(Suplemento 1), S487-S500.

Cortés, J. (1996). Comunidades coralinas y arrecifes del Área de Conservación Guanacaste, Costa Rica. Revista de Biología Tropical, 44(3), 623-625.

Cortés, J. (2017). Marine biodiversity baseline for Área de Conservación Guanacaste, Costa Rica: published records. ZooKeys, 652, 129-179.
Cortés, J. (2019). Capítulo 26: Isla del Coco, Costa Rica, Eastern Tropical Pacific. In Y. Loya, K. Puglise, \& T. Birdge (Eds.), Mesophotic Coral Ecosystems (pp. 465-475). Cham, Switzerland: Springer Nature.

Cortés, J., \& Joyce, F. (2020). BioMar-ACG: A successful partnership to inventory and promulgate marine biodiversity. Biotropica, 52, 1104-1107.

Deichmann, E. (1941). Coelenterates collected on the Presidential cruise of 1938. Smithsonian Miscellaneus Collection, 99, 1-17.

Etnoyer, P., \& Morgan, L. E. (2005). Habitat-forming deep-sea corals in the Northeast Pacific Ocean. In A. Freiwald, \& J. M. Roberts (Eds.), Cold-water Corals and Ecosystems (pp. 331-343). Erlangen Earth Conference Series. Berlin, Heilderberg: Springer.

Excoffon, A. C., Acuña, F. H., \& Cortés, J. (2009). The sea anemone Nemanthus californicus (Cnidaria: Actiniaria: Nemanthidae) from Costa Rica: re-description and first record outside the type locality. Marine Biodiversity Records, 2, 1-5.

Gori, A., Grinyó, J., Dominguez-Carrió, C., Ambroso, S., López-González, P. J., Gili, J. M., \& Bo, M. (2019). Deep gorgonian and black coral assemblages in the Mediterranean Sea. In C. Orejas, \& C. Jiménez (Eds.), Mediterranean Cold-Water Corals: Past, Present and Future (pp. 245-248). Coral Reefs of the World, 9. New York: Springer International Publishing.

Gress, E., \& Kaimuddin, M. (2021). Observations of sea anemones (Hexacorallia: Actiniaria) overgrowing black corals (Hexacorallia: Antipatharia). Marine Biodiversity, 51(3), 1-5.

Grigg, R. W. (2001). Black coral: History of a sustainable fishery in Hawai'i. Pacific Science, 55(3), 291-299.

Hickman, C. P. (2008). A Field Guide to Corals and Other Radiates of Galapagos: An Illustrated Guidebook to the Corals, Anemones, Zoanthids, Balck Corals, Gorgonians, Sea Pens, and Hydroids of the Galapagos Islands. Lexington, Virginia: Sugar Spring Press.

Love, M. S., Yoklavich, M. M., Black, B. A., \& Andrews, A. H. (2007). Age of black coral (Antipathes dendrochristos) colonies, with notes on associated invertebrate species. Bulletin of Marine Science, $80(2)$, 391-399.

Milne-Edwards, H., \& Haime, J. (1857). Histoire Narurelle des Coralliaires: ou polypes proprement dits. Paris: Librairie Encyclopédique de Roret.

Opresko, D. M. (1976). Redescription of Antipathes panamensis Verrill (Coelenterata, Antipatharia). Pacific Science, 30, 235-240.

Opresko, D. M. (2009). A new name for the Hawaiian Antipatharian coral formerly known as Antipathes 
dichotoma (Cnidaria: Anthozoa: Antipatharia). Pacific Science, 63(2), 277-291.

Opresko, D. M., \& Breedy, O. (2010). A new species of antipatharian coral (Cnidaria: Anthozoa: Antipatharia: Schizopathidae) from the Pacific coast of Costa Rica. Proceedings of the Biological Society of Washington, 123, 234-241.

Ortiz-Malavasi, E. (2014). Atlas de Costa Rica 2014. https://repositoriotec.tec.ac.cr/handle/2238/6749

Prouty, N. G., Roark, E. B., Buster, N. A., \& Ross, S. W. (2011). Growth rate and age distribution of deep-sea black corals in the Gulf of Mexico. Marine Ecology Progress Series, 423, 101-115.

QGIS Development Team. (2016). QGIS geographic information system. Open Source Geospatial Foundation Project. http://qgis.org

Raimundo, J., Vale, C., Caetano, M., Anes, B., CarreiroSilva, M., Martins, I., \& Porteiro, F. M. (2013). Element concentrations in cold-water gorgonians and black coral from Azores region. Deep Sea Research Part II: Topical Studies in Oceanography, 98, 129-136.

Reimer, J., \& Fujii, T. (2010). Four new species and one new genus of zoanthids (Cnidaria, Hexacorallia) from the Galápagos Islands. ZooKeys, 42, 1-36.
Roark, E. B., Guilderson, T. P., Dunbar, R. B., Fallon, S. J., \& Mucciarone, D. A. (2009). Extreme longevity in proteinaceous deep-sea corals. Proceedings of the National Academy of Sciences USA, 106(13), 5204-5208.

Vargas, R. (2000). Periclimenies murcielagensis, a new species of shrimp (Crustacea: Decapoda: Palaemonidae) living on black coral from the Pacific coast of Costa Rica. Proceedings of the Biological Society of Washington, 113, 17-23.

Verrill, A. E. (1869). Review of the corals and polyps of the west coast of America. Transactions of the Connecticut Academy of Arts and Science, 1, 377-567. (Black corals, 499-500).

Wagner, D., Luck, D. G., \& Toonen, R. J. (2012). The biology and ecology of black corals (Cnidaria: Anthozoa: Hexacorallia: Antipatharia). Advances in Marine Biology, 63, 67-132.

Wills-López, M. A. (2008). El coral negro Antipathes galapagensis en el complejo insular de Espíritu Santo, BCS, México. Ecología poblacional e historia de su explotación (Tesis de Maestría). Centro de Investigaciones Biológicas de Noroeste, La Paz, Baja California Sur, México. 